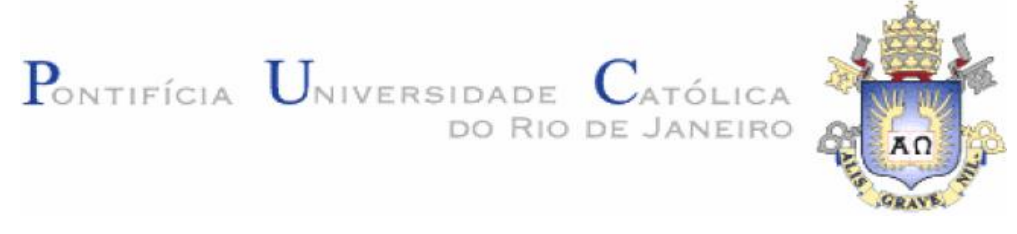

Constantino Niño Pinto

Uso de Elementos Discretos na Modelagem Numérica da Perfuração de Poços de Petróleo por Brocas PDC

Dissertação apresentada como requisito parcial para obtenção do título de Mestre pelo Programa de PósGraduação em Engenharia Civil da PUC-Rio

Orientador: Prof. Sergio Augusto Barreto da Fontoura 
Constantino Niño Pinto

\section{Uso de Elementos Discretos na Modelagem Numérica da Perfuração de Poços de Petróleo por Brocas PDC}

Dissertação apresentada como requisito parcial para obtenção do título de Mestre pelo Programa de PósGraduação em Engenharia Civil da PUC-Rio. Aprovada pela Comissão Examinadora abaixo assinada.

Prof. Sergio Augusto Barreto da Fontoura.

Orientador

PUC-Rio

Prof. Eurípedes do Amaral Vargas jr. PUC-Rio

Prof. Celso Kazuyuki Morooka

UNICAMP

Dr. Nelson Inoue

GTEP/PUC-Rio

Prof. José Eugênio Leal Coordenador Setorial do Centro

Técnico Científico - PUC-Rio

Rio de Janeiro, 25 de fevereiro de 2011 
Todos os direitos reservados. É proibida a reprodução total ou parcial do trabalho sem autorização da universidade, do autor e do orientador.

\section{Constantino Niño Pinto}

Graduou-se em Engenharia Civil na Universidade Industrial de Santander UIS (Bucaramanga, Santander, Colômbia - 2007). Trabalho no campo estrutural na área da indústria privada da construção. No ano 2009 ingressou ao curso de Mestrado em Engenharia Civil na Pontifícia Universidade Católica do Rio de Janeiro, na área de Geotécnica, desenvolvendo dissertação de mestrado na linha de pesquisa Geomecânica do Petróleo.

Ficha Catalográfica

Pinto, Constantino Niño

Uso de Elementos Discretos na Modelagem Numérica da Perfuração de Poços de Petróleo por Brocas PDC / Constantino Niño Pinto; Orientador: Sérgio A. B. da Fontoura - 2011.

142 f. : il. (color.) ; $30 \mathrm{~cm}$

Dissertação (mestrado) - Pontifícia Universidade Católica do Rio de Janeiro, Departamento de Engenharia Civil, 2011.

Incluí bibliografia

1. Engenharia Civil - Tese. 2. PDC. 3. Perfuração. 4. Método dos Elementos Discretos. 5. PDC2D. 6. Corte em rocha. 7. Ensaio de Corte em Rocha. 8. Energia mecânica específica. I. Fontoura, Sérgio A. B. da. II. Pontifícia Universidade Católica do Rio de Janeiro. Departamento de Engenharia Civil. III. Título.

CDD: 624 
Aos meus pais, 


\section{Agradecimentos}

A meus pais e meus irmãos, pelo amor e carinho.

Ao Professor Sergio A. B. Fontoura, pela sua orientação e ajuda no desenvolvimento desta tese.

Ao Nelson Inoue, pela colaboração, motivação, ajuda e apoio.

Ao GTEP pela infraestrutura e suporte.

À Baker Hughes pelo apoio financeiro.

À Coordenação de Aperfeiçoamento de Pessoal de Nível Superior (CAPES) pelo apoio financeiro.

Ao Departamento de Engenharia Civil da PUC-Rio pela colaboração. 


\section{Resumo}

Pinto, Constantino Niño; Fontoura, Sergio Augusto Barreto. Uso de Elementos Discretos na Modelagem Numérica da Perfuração de Poços de Petróleo por Brocas PDC. Rio de Janeiro, 2010. 142p. Dissertação de Mestrado - Departamento de Engenharia Civil. Pontifícia Universidade Católica do Rio de Janeiro.

Perfuração em rochas é analisada através de métodos empíricos não havendo uma metodologia racional aceita para analisar todos os parâmetros que controlam a destruição das rochas. Esta dissertação tem como objetivo analisar a perfuração de rochas do ponto de vista de modelagem numérica, utilizando o método dos elementos distintos. Inicialmente será discutida a modelagem de rochas, um meio quase-contínuo, por um conjunto de partículas cimentadas. Em seguida, a destruição da rocha por brocas de perfuração será simulada analisando o processo de corte em rocha usando um único cortador. O produto final desta dissertação é comparar resultados de energia mecânica especifica obtidos em ensaios em laboratório com os resultados da modelagem e concluir a eficiência desta metodologia. Além disto, será possível propor estudos paramétricos para avaliar a importância de alguns elementos geométricos no ambiente de corte para melhorar a eficiência da operação.

\section{Palavras-chave}

PDC; Perfuração; Método dos Elementos Discretos; PFC2D; Corte em rocha; Ensaio de Corte em Rocha; Energia mecânica específica. 


\section{Abstract}

Pinto, Constantino Niño; Fontoura, Sergio Augusto Barreto (Advisor). Use to Distinct Elements in the Drilling Numerical Modeling of Petroleum Wells by PDC bits. Rio de Janeiro, 2010. 142p. MsC Dissertation Departamento de Engenharia Civil, Pontifícia Universidade Católica do Rio de Janeiro.

Drilling in rock is analyzed through empirical methods and there is none accepted rational methodology to analyze all the parameters that control the destruction of rocks. This dissertation aims to analyze the drilling of rocks from the standpoint of numerical modeling, using the Distinct Elements Method. Initially we discuss the modeling of rocks, a quasi-continuous medium, by a set of bonded particles. Then, the destruction of rock by drill bits will be simulated by analyzing the rock cutting process using a single cutter. The final product of this work is to compare results of specific mechanical energy obtained in laboratory tests with modeling results and conclude the method's efficiency. Moreover it is possible to propose parametric studies to evaluate the importance of some geometric elements in the cut environment to improve efficiency of the operation.

\section{Keywords}

PDC; Drilling; Distinct Element Method; PFC2D; Rock Cutting; Scratch Test; Mechanical Specific Energy. 


\section{Sumário}

1 Introdução 21

1.1. Definição do Problema 21

1.2. Objetivo do trabalho 23

1.3. Escopo do trabalho 25

2 Revisão Bibliográfica $\quad 26$

2.1. Introdução 26

2.2. Ensaios experimentais 26

2.2.1. O ensaio de corte como uma medida de resistência para rochas sedimentares. 26

2.2.2. Influência da poropressão na perfuração de rochas com baixa permeabilidade e com dilatância. 30

2.2.3. Dependência da energia especifica na perfuração de poços profundos em folhelhos. 32

2.2.4. Estudo experimental de corte em rocha sob pressão hidrostática. 35

2.2.5. Estudo experimental da MSE usando um cortador simples sob condições simuladas de pressão. 43

2.3. Ensaios Numéricos. 49

2.3.1. Modelagem usando PFC2D de corte em rocha sob altas condições de pressão. $\quad 49$

2.3.2. Rol do modo de fratura na dinâmica do corte em rocha. 54

2.3.3. Simulação do corte em rocha usando o método dos elementos distintos - PFC2D. 58

3 Teoria do Método dos Elementos Discretos. 65

3.1. Introdução 65

3.2. Formulação do Método dos Elementos Discretos. 67

3.3. Lei Força-Deslocamento 68

3.4. Lei de Movimento 73

3.5. Modelo de contato $\quad 74$

3.6. Parâmetros na modelagem de ensaios triaxiais 77 
4 Modelagem de ensaios triaxiais. $\quad 79$

4.1. Introdução $\quad 79$

4.2. Preparação da Amostra 80

4.3. Carregamento quasi-estático 81

4.4. Efeito da discretização. 83

4.5. Ensaios Numéricos à Compressão. 84

4.5.1. Genesis do material 84

4.5.2. Procedimento para o refinamento do tamanho da partícula: 86

4.5.3. Definindo a densidade das partículas. 87

4.5.4. Ensaio à compressão. 88

4.5.5. Esquemas de medição de tensão e deformação. 89

4.5.6. Taxa de Carregamento 89

4.5.7. Descrição do ensaio à compressão. 90

4.6. Obtenção dos Micro-parâmetros usando ensaios à compressão. 93

5 Corte em Rocha $\quad 97$

$\begin{array}{ll}\text { 5.1. Introdução } & 97\end{array}$

5.2. Metodologia 98

5.3. Cálculos, simulação do Corte e resultados 103

5.3.1. Corte sob condições atmosféricas: 104

5.3.2 Corte sob pressão confinante 109

6 Conclusões e Sugestões 134

6.1. Conclusões 134

$\begin{array}{ll}\text { 6.2. Sugestões } & 137\end{array}$

Referências Bibliográficas 139 


\section{Lista de figuras}

Figura 1.1. (a) Broca PDC e (b) esquema de um cortador simples para uma broca PDC. (Hareland et al., 2007)

Figura 2.1. Corte no Arenito de Berea. (a) Modo dúctil para $\mathrm{d}=1 \mathrm{~mm}$ (b) e modo frágil para d = $4 \mathrm{~mm}$. (Richard et al., 1998)

Figura 2.2. Correlação entre a energia específica intrínseca e a resistência uniaxial à compressão. (Richard et al. 1998)

Figura 2.3. Ensaios executados usando o Sharp cutter para arenito de Red Wildmoor. (Richard et al., 1998)

Figura 2.4. Perfil de energia especifica obtido usando o cortador perfeito com $1,00 \mathrm{~mm}$ de profundidade de corte em rocha de reservatório. (Richard et al., 1998)

Figura 2.5. Configuração do processo de corte em rocha. (Detournay et al., 2000)

Figura 2.6. Energia mecânica específica em função da pressão de confinamento no caso de corte em rocha usando o folhelho de Mancos. (Zijsling, 1987)

Figura 2.7. Energia mecânica específica em função da resistência a compressão simples no caso de corte em rocha usando o folhelho de Mancos. (Zijsling, 1987)

Figura 2.8. Célula Triaxial ATC com dispositivo de corte. (Detournay, 2002) 33

Figura 2.9. Variação da energia específica em função da poropressão inicial para Johnstone e para o folhelho de Pierre I, com pressão confinante de 30 MPa e ângulo de ataque de $15^{\circ}$. (Detournay, 2002)

Figura 2.10. Variação da energia especifica em função à pressão de confinamento para Johnstone e os folhelhos de Pierre I e Mancos. (Detournay, 2002)

Figura 2.11. Vista superior da configuração do equipamento. (Kaitkay et al., 2002)

Figura 2.12. Geometria da ferramenta de corte. (Kaitkay et al., 2002)

Figura 2.13. Ilustração das três posições de corte para cada teste. (Kaitkay, 2002)

Figura 2.14. Configuração mecânica experimental do corte em pressão confinante. (Kaitkay, 2002) 
Figura 2.15. Ferramenta de corte para testes em pressão confinante (Kaitkay, 2002)

Figura 2.16. Variação das forças no cortador em função do tempo em pressão atmosférica. (Kaitkay, 2002)

Figura 2.17. Média das forças no corte pressão atmosférica. (Kaitkay, 2002). 40

Figura 2.18. Variação das forças no cortador em função do tempo em pressão confinante. (Kaitkay, 2005).

Figura 2.19. Variação da média das forças no corte em pressão confinante (Kaitkay, 2002).

Figura 2.20. Mecanismo do material removido durante o corte. (Kaitkay, 2002).

Figura 2.21. Célula de alta pressão da universidade de Tulsa (Rafatian, 2009).

Figura 2.22. Esquema de montagem do equipamento de corte (Garcia, 1998) 44

Figura 2.23. Forças no cortador e suas direções (Rafatian, 2009).

Figura 2.24. Mármore de Cartago na esquerda, e Calcário de Indiana na direita depois do teste de corte (Rafatian, 2009).

Figura 2.25. MSE VS. Pressão confinante para o mármore de Cartago em óleo leve e óleo viscoso (Rafatian, 2009).

Figura 2.26. Eficiência de corte versus pressão confinante para o mármore de Cartago com óleo leve e óleo viscoso (Rafatian, 2009).

Figura 2.27. Curvas tensão deformação geradas de rochas virtuais PFC.

(Ledgerwood III, 2009).

Figura 2.28. Típicos espécimes triaxiais. Esquerda 6.90 MPa de pressão confinante, direita $280 \mathrm{MPa}$ de pressão confinante. (Ledgerwood III, 2009)

Figura 2.29. Corte a pressão atmosférica. (Ledgerwood III, 2009)

Figura 2.30. Corte a pressão de 20 Mpa. (Ledgerwood III, 2009)

Figura 2.31. Calibração biaxial. (a) curva tensão deformação para Piceance Shale, e (b) envoltória de falha para Berea Sandstone. (Block, 2009). 55

Figura 2.32. Ambiente de corte em PFC2D. (Block, 2009).

Figura 2.33. Diagrama de densidade $\left(\right.$ watt $\left./ \mathrm{m}^{2}\right)$ para $\mathrm{WOB}=30 \mathrm{KN}$ e Pm $=0$

MPa. (Block, 2009).

Figura 2.34. Diagrama de densidade $\left(w a t t / \mathrm{m}^{2}\right)$ para $\mathrm{WOB}=200 \mathrm{KN}$ e $\mathrm{Pm}=30$ MPa. (Block, 2009).

Figura 2.35. MSE como resultado das simulações de corte em rocha. (Block, 
2009).

Figura 2.36. Energia no modo dúctil em função da profundidade. (Block, 2009).

Figura 2.37. Espécime de rocha virtual. (Lei, 2004).

Figura 2.38. Sem pressão confinante. (Lei et al. 2004).

Figura 2.39. Com pressão confinante de $34.4 \mathrm{MPa}$. (Lei et al. 2004).

Figura 2.40. Forças no caso sem pressão confinante. (Lei et al. 2004).

62

Figura 2.41. Forças no caso com pressão confinante. (Lei et al. 2004).

62

Figura 2.42. Variação da média da força em função da profundidade de corte e do ângulo de ataque. (Lei et al. 2004).

Figura 2.43. Variação da média da força em função da pressão confinante.

(Lei et al. 2004).

Figura 3.1. Ciclo de cálculo do método dos elementos discretos, (Ayquipa, 2008)

Figura 3.2. Nomenclatura partícula - partícula. (Itasca, 2008)

Figura 3.3. Nomenclatura partícula - parede. (Itasca, 2008)

Figura 3.4. Ligação paralela descrita como uma peça de tamanho finito do material cimentante. (Itasca, 1999)

Figura 4.1. Envoltória de ruptura biaxial no plano o1-б3, (Huang, 1999)

Figura 4.2. Comportamento da tensão normal $\sigma 0$ para uma porosidade qualquer num arranjo de partículas com $\mathrm{Kn} /(\mathrm{Ks}=1)$, (Huang, 1999)

Figura 4.3. Curva esquemática para o ensaio uniaxial. (Huang, 1999)

Figura 4.4. Variação da resistência à compressão uniaxial $\sigma c$ com a relação LR. (Huang, 1999)

Figura 4.5. Procedimento da gênesis do material. (a) arranjo de partículas inicialmente gerado; (b) forças de contato distribuídas depois do segundo passo; (c) partículas flutuantes e contatos depois do passo 2; (d) rede de ligação paralela depois do passo 4.

Figura 4.6. Curva Tensão - Deformação do ensaio Triaxial numérico para o mármore de Cartago com uma pressão confinante de 34,4 MPa em PFC2d.

Figura 4.7. Curva Tensão - Deformação do ensaio Triaxial numérico para o mármore de Cartago com uma pressão confinante de 3,44 MPa em PFC2d.

Figura 5.1. Valores de força típicos no ensaio de corte em rocha em arenito de Voges com $d=3,6 \mathrm{~mm}$ e $\theta=15^{\circ}$. (Richard, 1999). 
Figura 5.2. Ambiente de corte. (Itasca, 1999). 99

Figura 5.3. Configuração do processo de corte. (Huang, 1999) 99

Figura 5.4. Ambiente de corte simulado em PFC2d para condições atmosféricas.

Figura 5.5. Ambiente de corte simulado em PFC2d para condições sob pressão confinante.

Figura 5.6. Principais componentes do ambiente de corte simulado em PFC2d.

Figura 5.7. Forças no cortador $(\mathrm{N})$ vs deslocamento da ferramenta de corte $(\mathrm{m})$.

Figura 5.8. Ambiente de corte para condições atmosféricas para $25 \mathrm{~mm}$

Figura 5.9. Detalhe do corte para condições atmosféricas para $25 \mathrm{~mm}$ de deslocamento da ferramenta de corte

Figura 5.10. Forças no cortador em $(\mathrm{N})$ para condições atmosféricas vs Deslocamento da ferramenta de corte em $(\mathrm{m})$

Figura 5.11. Trabalho mecânico acumulado em $(\mathrm{kN}-\mathrm{m} / \mathrm{m})$ para condições atmosféricas vs deslocamento da ferramenta de corte em $(\mathrm{m})$

Figura 5.12 MSE para diferentes valores de ângulo de ataque sob pressão atmosférica.

Figura 5.13 MSE para diferentes valores de profundidade de corte sob condições atmosféricas.

Figura 5.14 Forças no cortador com 2,80 MPa de pressão confinante para $25 \mathrm{~mm}$

Figura 5.15 Detalhe do corte para 2,80 MPa de pressão confinante para $25 \mathrm{~mm}$ de deslocamento da ferramenta de corte

Figura 5.16. Forças no cortador em $(\mathrm{N})$ para pressão de confinamento de 2.80 MPa vs deslocamento da ferramenta de corte em $(\mathrm{m})$

Figura 5.17. Trabalho mecânico acumulado em (kN-m/m) para 2,80 MPa de pressão de confinamento vs deslocamento da ferramenta de corte em $(m)$

Figura 5.18. Forças no cortador com 6,65 MPa de pressão confinante para $25 \mathrm{~mm}$

Figura 5.19. Detalhe do corte para 6,65 MPa de pressão confinante para $25 \mathrm{~mm}$ de deslocamento da ferramenta de corte

Figura 5.20. Forças no cortador em $(\mathrm{N})$ para pressão confinante de 6.65 MPa vs deslocamento da ferramenta de corte em (m) 
Figura 5.21. Trabalho mecânico acumulado em $(\mathrm{kN}-\mathrm{m} / \mathrm{m})$ para 6,65 MPa de pressão de confinamento vs deslocamento da ferramenta de corte em $(m)$

Figura 5.22. Forças no cortador com 34,4 MPa de pressão confinante para $25 \mathrm{~mm}$

Figura 5.23. Detalhe do corte para 34,4 MPa de pressão confinante para $25 \mathrm{~mm}$ de deslocamento da ferramenta de corte

Figura 5.24. Forças no cortador em (N) para pressão confinante de $34.4 \mathrm{MPa}$ vs Deslocamento da ferramenta de corte em $(\mathrm{m})$

Figura 5.25. Trabalho mecânico acumulado em $(\mathrm{kN}-\mathrm{m} / \mathrm{m})$ para 34,4 MPa de pressão de confinamento vs deslocamento da ferramenta de corte em (m)

Figura 5.26. Forças no cortador com $60 \mathrm{MPa}$ de pressão confinante para $25 \mathrm{~mm}$

Figura 5.27 Detalhe do corte para $60 \mathrm{MPa}$ de pressão confinante para $25 \mathrm{~mm}$ de deslocamento da ferramenta de corte

Figura 5.28. Forças no cortador em (N) para pressão confinante de $60.0 \mathrm{MPa}$ vs deslocamento da ferramenta de corte em $(\mathrm{m})$

Figura 5.29 Trabalho mecânico acumulado em $(\mathrm{kN}-\mathrm{m} / \mathrm{m})$ para $60 \mathrm{MPa}$ de pressão de confinamento vs deslocamento da ferramenta de corte em (m)

Figura 5.30. Forças no cortador com $90 \mathrm{MPa}$ de pressão confinante para $25 \mathrm{~mm}$

Figura 5.31. Detalhe do corte para $90 \mathrm{MPa}$ de pressão confinante para $25 \mathrm{~mm}$ de deslocamento da ferramenta de corte

Figura 5.32. Forças no cortador em (N) para pressão confinante de $90.0 \mathrm{MPa}$ vs deslocamento da ferramenta de corte em $(\mathrm{m})$

Figura 5.33. Trabalho mecânico acumulado em $(\mathrm{kN}-\mathrm{m} / \mathrm{m})$ para $90 \mathrm{MPa}$ de pressão de confinamento vs deslocamento da ferramenta de corte em $(m)$

Figura 5.34. Forças no cortador com $120 \mathrm{MPa}$ de pressão confinante para $25 \mathrm{~mm}$

Figura 5.35. Detalhe do corte para $120 \mathrm{MPa}$ de pressão confinante para $25 \mathrm{~mm}$ de deslocamento da ferramenta de corte

Figura 5.36. Forças no cortador em (N) para pressão confinante de $120.0 \mathrm{MPa}$ vs deslocamento da ferramenta de corte em $(\mathrm{m})$ 
Figura 5.37. Trabalho mecânico acumulado em (kN-m/m) para 120 MPa de pressão de confinamento vs deslocamento da ferramenta de corte em $(m)$

Figura 5.38. MSE vs Pressão Confinante para $25 \mathrm{~mm} \quad 128$

Figura 5.39. MSE vs Pressão confinante para $25 \mathrm{~mm} \quad 129$

Figura 5.40. Força média no cortador vs Pressão confinante para $10 \mathrm{~mm} \quad 130$

Figura 5.41. MSE vs Pressão confinante para $25 \mathrm{~mm}$ de corte

Figura 5.42. MSE para diferentes valores de ângulo de ataque com pressão confinante de $34,4 \mathrm{MPa}$

Figura 5.43. MSE para diferentes valores de profundidade de corte com pressão confinante de $34,4 \mathrm{MPa}$. 


\section{Lista de tabelas}

Tabela 2.1. Correlação entre a energia especifica e a resistência uniaxial a compressão. (Richard et al. 1998)

Tabela 2.2. Condições mecânicas para o corte em pressão atmosférica.

(Kaitkay, 2002)

Tabela 2.3. Condições mecânicas para o corte em pressão confinante.

(Kaitkay, 2002)

Tabela 2.4. Propriedades da amostra de rocha (Rafatian, 2009).

Tabela 2.5. Principais parâmetros para simular o mármore de Cartago. (Lei, 2004).

Tabela 2.6. Resultados dos ensaios numéricos biaxiais. (Lei et al. 2004).

Tabela 2.7. Condições da simulação do processo de corte. (Lei, 2004).

Tabela 3.1. Nomenclatura partícula - parede. (Itasca, 2008)

Tabela 4.1. Parâmetros para a geração do espécime.

Tabela 4.2. Comparação de resultados entre as propriedades reais do mármore de Cartago e a simulação do ensaio a compressão.

Tabela 5.1. Micro - parâmetros usados na preparação da amostra para caracterizar o mármore de Cartago. 101

Tabela 5.2. Resultados da simulação para corte sob pressão atmosférica. 106

Tabela 5.3. MSE para diferentes valores do ângulo de ataque em condições atmosféricas.

Tabela 5.4. MSE para diferentes valores de profundidade de corte em condições atmosféricas.

Tabela 5.5. Resultados da simulação para corte em rocha com pressão confinante de 2,80 MPa.

Tabela 5.6. Resultados da simulação para corte em rocha com pressão confinante de 6,65 MPa.

Tabela 5.7. Resultados da simulação para corte em rocha com pressão confinante de 34,4 MPa.

Tabela 5.8. Resultados da simulação para corte em rocha com pressão confinante de $60 \mathrm{MPa}$.

Tabela 5.9. Resultados da simulação para corte em rocha com pressão confinante de $90 \mathrm{MPa}$.

Tabela 5.10. Resultados da simulação para corte em rocha com pressão 
confinante de $120 \mathrm{MPa}$.

Tabela 5.11. Resultados de CCS para diferentes pressões confinantes, Terra Tek, 2007.

Tabela 5.12. Resultados da simulação do ensaio de corte para diferentes pressões confinantes para $25 \mathrm{~mm}$

Tabela 5.13. MSE para diferentes valores do ângulo de ataque em sob pressão confinante de $34,4 \mathrm{MPa}$.

132

Tabela 5.14. MSE para diferentes valores de profundidade de corte sob pressão confinante de $34,4 \mathrm{MPa}$. 


\section{Lista de Símbolos}

$\alpha$

$\alpha^{1}$

$C_{k}$

$d_{i j}$

$\Delta \varepsilon_{x}$

$\Delta \varepsilon_{y}$

$\Delta \sigma_{y}$

$\Delta t$

$\Delta \mathrm{U}_{i}^{S}$

$e_{i j k}$

E

$\varepsilon_{t}$

$\dot{\varepsilon}_{p}$

$f$

F

$F_{x}$

$F_{y}$

$F_{i}$

$F_{i}^{n}$

$F_{i}^{S}$

$g_{i}$

$H_{i}$

I

$K_{n}$

$K_{S}$

$\lambda$

$\zeta$

$L$

$L_{o}$

m

$M_{i}^{[a j]}$

MSE
Inclinação do plano de cisalhamento

Delta de Kronecker

Produto vetorial das normais $n_{m}^{[O L D]}$ e $n_{n}$

Distância entre os centros de duas partículas $i$ e $j$.

Diferencial de deformação em $x$

Diferencial de deformação em $y$

Diferencial de tensão em $y$

Passo de tempo

Deslocamento cisalhante

Símbolo de permutação

Modulo de Young

Tolerância do mecanismo de servo-controle

Taxa de deformação específica biaxial

Taxa de penetração

Força total de corte

Força no cortador na direção do corte

Força no cortador na direção perpendicular ao corte

Força total aplicada no contato $i$

Força normal aplicada no contato $i$

Força cisalhante aplicada no contato $i$

Gravidade atuando na partícula $i$

Momento angular na partícula $i$

Momento de inércia da partícula

Rigidez normal da partícula

Rigidez cisalhante da partícula

Multiplicador do raio do contato na ligação paralela

Tangente geométrica do ângulo de corte e de atrito da rocha

Comprimento do meio discreto

Altura do espécime biaxial

Massa de uma partícula

Momento aplicado na partícula $a j$

Energia Mecânica Especifica 


\begin{tabular}{|c|c|}
\hline$n_{i}$ & Vetor que conecta os centros de duas partículas \\
\hline$n_{m}^{[O L D]}$ & Vetor normal do passo de tempo anterior \\
\hline$n_{n}$ & Vetor normal do passo de tempo atual \\
\hline$\varphi$ & Ângulo de atrito da rocha \\
\hline$\psi$ & Ângulo da interface rocha-cortador \\
\hline$\rho$ & Densidade do meio discreto \\
\hline$\rho_{b}$ & Densidade de uma partícula \\
\hline$P_{o}$ & Poropressão \\
\hline$P_{m}$ & Pressão confinante \\
\hline$P_{b}$ & Pressão interna \\
\hline$P_{c}^{t}$ & Pressão confinante do mecanismo de servo-controle \\
\hline$\theta$ & Ângulo de ataque \\
\hline$q$ & Resistência à compressão simples \\
\hline$R$ & Raio médio de um arranjo de partículas \\
\hline$R^{[\alpha]}$ & Raios de uma partícula $\alpha$ \\
\hline$R O P$ & Taxa de perfuração \\
\hline$R P M$ & Revoluções por minuto \\
\hline$\sigma_{1}$ & Tensão principal máxima \\
\hline$\sigma_{3}$ & Tensão principal mínima \\
\hline $\bar{\sigma}_{c}$ & Resistência normal do contato \\
\hline$\sigma_{f}$ & Resistência pico \\
\hline$\sigma_{c i}$ & Tensão do inicio das trincas \\
\hline$\sigma_{0}^{t}$ & Tensão isotrópica especifica \\
\hline$\sigma_{c}$ & Tensão confinante \\
\hline$\sigma_{\text {ref }}$ & Tensão de referência \\
\hline$\sigma_{\text {num }}$ & Tensão axial numérica máxima \\
\hline$\sigma_{\text {sat }}$ & Solução analítica da tensão axial \\
\hline$\sigma_{a}^{t}$ & Tensão axial do mecanismo de servo-controle \\
\hline$\sigma_{d}$ & Tensão desviadora \\
\hline$s$ & Comprimento do corte \\
\hline $\bar{\tau}_{c}$ & Resistência ao cisalhamento do contato \\
\hline$U^{n}$ & Sobreposição entre duas partículas \\
\hline$v$ & Velocidade linear \\
\hline$v_{p}$ & Velocidade final de carregamento de servo-controle \\
\hline$v$ & Coeficiente de Poisson \\
\hline
\end{tabular}




$\begin{array}{ll}V & \text { Velocidade do corte } \\ V^{(P)} & \text { Volume da partícula } P \\ V_{i} & \text { Velocidade na partícula } i \\ V_{i}^{S} & \text { Componente cisalhante da velocidade numa partícula } \\ V_{i}^{n} & \text { Componente normal da velocidade numa partícula } \\ \omega_{i} & \text { Velocidade angular da partícula } i \\ \dot{\omega}_{i} & \text { Aceleração angular da partícula } i \\ W O B & \text { Peso sobre a broca } \\ x_{i} & \text { Posição da partícula } i \\ \dot{x}_{i} & \text { Velocidade da partícula } i \\ \ddot{x}_{i} & \text { Aceleração da partícula } i \\ \dot{x}_{i}[a j] & \text { Velocidade translacional da partícula } a j \\ x_{i}^{[c]} & \text { Posição do contato } c\end{array}$

